Check for updates

Cite this: RSC Adv., 2017, 7, 28105

Received 26th March 2017

Accepted 12th May 2017

DOI: $10.1039 / c 7 r a 03499 g$

rsc.li/rsc-advances

\title{
Boosted adsorption-photocatalytic activities and potential lithium intercalation applications of layered potassium hexaniobate nano-family $\dagger$
}

\author{
Qinglin Deng, Mengjiao Li, Junyong Wang, Peng Zhang, Kai Jiang, Jinzhong Zhang, \\ Zhigao Hu iD * and Junhao Chu
}

\begin{abstract}
Two-dimensional layered $\mathrm{K}_{4} \mathrm{Nb}_{6} \mathrm{O}_{17}(\mathrm{KN})$ possesses two different types of interlayer regions, which is of great interest for applications in energy conversion, environmental purification, etc. Although the photocatalytic properties of KN have been extensively studied, there still remain some pivotal problems that need to be clarified for future applications. Here we demonstrate that the KN nano-family (including $\mathrm{KN}$ nanolaminas and nano hollow spheres) can be derived from the same $\mathrm{Nb}_{2} \mathrm{O}_{5}$-based hydrothermal reaction. Different morphologies of $\mathrm{KN}$ show unique microstructures and optoelectronic properties. Remarkably, the initial $\mathrm{pH}$ of a dye solution has been proven to play a vital role in affecting the adsorption and photocatalytic performances of KN. Due to the effects of dye sensitization, KN shows superior photodegradation performance under both ultraviolet and visible light. Based on these crucial results, a highly-efficient and feasible scheme has been proposed to deal with dye wastewater. In addition, $\mathrm{KN}$ as a potential anode material for lithium ion batteries has been investigated for the first time. The present work could be helpful in broadening the multifunctional applications of KN and other layered niobate materials.
\end{abstract}

\section{Introduction}

Interest in expanding the practical applications of low dimensional semiconductor materials has surged in order to meet the needs of integrated systems, which can simultaneously realize various functions in different fields. ${ }^{\mathbf{1 - 4}}$ As a typical case, layered $\mathrm{K}_{4} \mathrm{Nb}_{6} \mathrm{O}_{17}$ (abbreviated as $\mathrm{KN}$ ) has attracted considerable attention and has been recognized as one of the most promising multifunctional materials owing to its charming features and widespread applications in energy conversion, environmental purification, catalysis, chemical intermediates, non-linear optics, etc. ${ }^{5-8} \mathrm{KN}$ is composed of octahedral units of $\mathrm{NbO}_{6}$ and possesses a typical two-dimensional (2D) layered structure via bridging oxygen atoms. There are two different types of interlayer regions, which have different properties in hydration and ion exchange. ${ }^{9-12}$

Most of the current research on $\mathrm{KN}$ is focused on its photochemical and ion exchange properties..$^{\mathbf{6 , 9 , 1 3 , 1 4}}$ At present, as an environmental issue, disposal of dye-based toxic effluents is considered to be one of the most challenging missions in

Technical Center for Multifunctional Magneto-Optical Spectroscopy (Shanghai), Department of Electronic Engineering, East China Normal University, Shanghai 200241, China. E-mail: zghu@ee.ecnu.edu.cn; Fax: +86-21-54345119; Tel: +86-2154345150

$\dagger$ Electronic supplementary information (ESI) available. See DOI: 10.1039/c7ra03499g making them environmentally friendly. ${ }^{4,15-18}$ Therefore, highlyefficient and multifunctional materials for dye degradation are urgently desired. ${ }^{19}$ As an effective and typical photocatalytic material, ${ }^{20-22} \mathrm{KN}$ can be synthesized by several methods, such as by conventional solid-state reaction, flux growth, sol-gel and hydrothermal methods. ${ }^{23-26}$ Among them, hydrothermal and sol-gel methods are well-accepted methods due to their evident advantages. ${ }^{24,27}$ On one hand, some researchers have prepared $\mathrm{KN}$ by hydrothermal methods, however, the niobium raw materials were incompletely used, which does not conform to the concept of green chemistry. So, it is significant to propose an approach for making full use of the raw materials. On the other hand, many studies have suggested that nano-materials with different morphologies would show distinctive performances. Therefore, preparing and investigating the photo/ electro-chemical properties of the $\mathrm{KN}$ nano-family could be an interesting issue. At this stage, although there are some reports on the good photodegradation properties of $\mathrm{KN},{ }^{7,28,29}$ its ability to adsorb dyes and the influence derived from the $\mathrm{pH}$ of the dye solution have been obviously ignored. Thus, these relevant kinetics mechanisms should be investigated in detail to further elucidate the photocatalytic performances of the $\mathrm{KN}$-based family and other similar layered semiconductor materials. As we know, like most other layered semiconductors, $\mathrm{KN}$ has a relatively large band gap (3.74 $\mathrm{eV}$ for $\mathrm{KN}$ nanolaminas in our work), with photocatalytic activities limited to ultraviolet (UV) light irradiation. Based on this, combining $\mathrm{KN}$ with 
a semiconductor which has a narrow band gap has frequently been employed to improve the efficient absorption of energy from sunlight. ${ }^{30-33}$ Fortunately, among them, lead sulfide (PbS) is a good modifier owing to its small band gap $(\sim 0.41 \mathrm{eV})$ and large exciton Bohr radius $(18 \mathrm{~nm}) .^{31,34}$ In addition, it was believed that $\mathrm{PbS}$ quantum dots (PbS QDs) could further improve the photocatalytic activity of KN. Additionally, to aid the global energy crisis, lithium ion batteries (LIBs) have been widely adopted as promising power sources for electric devices. ${ }^{35,36}$ Although there are numerous reports on anode materials for LIBs, novel materials with high energy and power densities have always been desired. ${ }^{37-39}$ As we know, just like titanium-based LIB anode materials, nanostructured niobiumbased oxides have emerged as one of the most promising materials for LIBs due to the rich redox chemistry of $\mathrm{Nb}^{\mathbf{4 0 - 4 3}}$ It is believed that layered $\mathrm{KN}$ should be considered as a potential anode active material due to its layered structure and because the redox of $\mathrm{Nb}^{5+} / \mathrm{Nb}^{4+}$ has the possibility to realize the reversible reaction with lithium. However, to the best of our knowledge, using KN for LIBs has not yet been reported.

In this work, much effort has been devoted to exploring the methodologies for preparing the KN nano-family (nanolaminas and nano hollow spheres) and investigating their relevant photo/electro-chemical properties. Remarkably, a novel and effective method based on a hydrothermal reaction is proposed to make full use of the raw materials. Moreover, systematic research of $\mathrm{KN}$ has shed light on the crucial effects of the initial $\mathrm{pH}$ of a dye solution on adsorption and photodegradation. These relevant kinetics mechanisms have been investigated in detail. In addition, we also researched its application in energy storage for LIBs. It is believed that the present work could be helpful in deeply understanding the intrinsic features of $\mathrm{KN}$ and developing potential multifunctional KN-based applications.

\section{Experimental section}

All of the chemical reagents and solvents used in the experiments were purchased from commercial sources and did not undergo further purification. The KN nanolaminas (KN-NL) and $\mathrm{KN}$ nano hollow spheres (KN-NS) were prepared by a mild hydrothermal process. The addition of appropriate amounts of urea plays a key role in the control of the morphology. KN-NL modified with PbS QDs (KN-NL@PbS) was prepared using 2mercaptopropionic acid as the surface-functionalized linker. The detailed synthesis processes and characterization methods are described in the ESI $\dagger$.

\section{Results and discussion}

\subsection{Crystal structure}

The X-ray diffraction (XRD) patterns of the powder samples are shown in Fig. 1(a) and S1 (ESI $\dagger$ ). It was found that the diffraction peaks of KN-NL and KN-NS can be assigned to the orthorhombic phase of $\mathrm{K}_{4} \mathrm{Nb}_{6} \mathrm{O}_{17}$ (JPCDS no. 76-0977). Note that $\mathrm{KN}$ has two different types of interlayer spaces alternately between the $\left(\mathrm{Nb}_{6} \mathrm{O}_{17}\right)^{4-}$ layers, which exhibit different intercalating

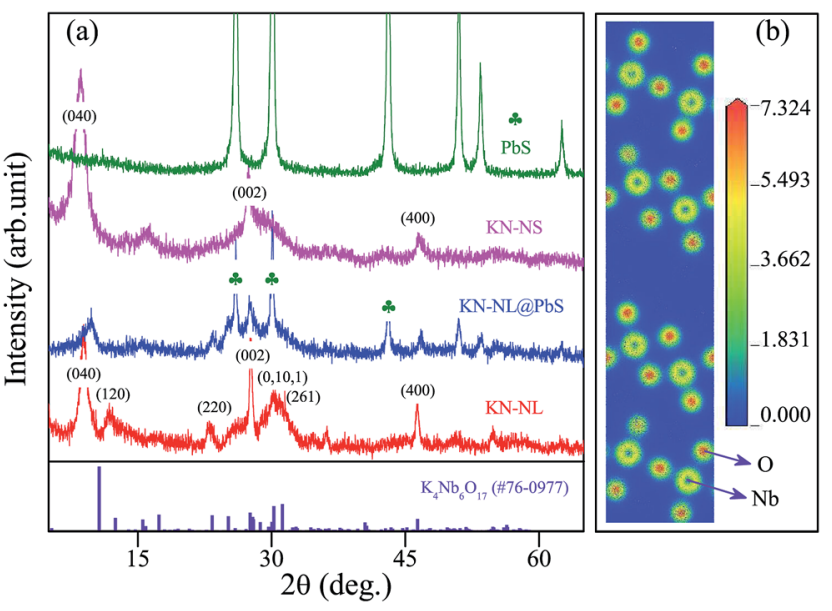

Fig. 1 (a) XRD patterns of powder samples including KN-NL, KN$\mathrm{NL} @ \mathrm{PbS}, \mathrm{KN}-\mathrm{NS}$ and $\mathrm{PbS}$ and (b) the charge density difference of KN.

capabilities, and that $\mathrm{KN}-x \mathrm{H}_{2} \mathrm{O}$ is easily formed by adsorbing moisture in air. ${ }^{10}$ The (040) diffraction peaks especially are always slightly different from the standard card. The $d_{040}$ spacing is the interlayer spacing of $\mathrm{KN}$, so it can be understood that the (040) diffraction peaks of hydrothermally-derived $\mathrm{KN}$ NL and KN-NS show a shift. The weak and broadening diffraction peaks for the $\mathrm{KN}$ powder samples suggest that the obtained products might be composed of nanoscale-sized crystal grains. Pristine PbS exhibits a strong and sharp characteristic reflection, which matches the PbS standard card (JPCDS no. 05-0592) well. As we can see, the diffraction peaks of the KN-NL@PbS composite were similar to those of pure KN-NL except for the peaks of $\mathrm{PbS}$, which indicated that the modification of $\mathrm{PbS}$ cannot destroy the KN structure. Moreover, Fig. S1 (ESI $\dagger$ ) reveals that pure $\mathrm{KNbO}_{3}$ and $\mathrm{KN}$ nanocrystalline films were also obtained. In addition, as shown in Fig. 1(b), the charge density difference of $\mathrm{KN}$ clearly depicts the strong interaction of $\mathrm{Nb}$ and $\mathrm{O}$ atoms due to the overlap of atomic orbitals.

\subsection{Surface morphologies and synthesis mechanisms}

The morphologies of the powder samples were characterized by scanning electron microscopy (SEM). As shown in Fig. 2(a)-(c) at different magnifications, the synthesized KN-NL exhibited aggregated lamina morphology. Correspondingly, the synthesis mechanism of KN-NL can be depicted as shown in Fig. 2(d). Firstly, the KN nanocrystals were generated after hydrothermal treatment, then they self-assembled to form nanolamina, and finally a certain amount of nanolamina aggregated together to reduce the potential energy of the surface. Diversely, it was found that KN-NS is spherical and that the average diameter of the hollow spheres is about $4 \mu \mathrm{m}$ (Fig. 2(e)-(g)). A closer observation indicates that the hollow spheres are composed of thin nanosheets, which pack densely and show a porous structure. The possible synthesis mechanism of KN-NS can be generalized in three steps, as depicted in Fig. 2(h): formation of fine nanocrystals and gas bubbles, attachment of the fine nanocrystals on the gas-liquid interface, and further aggregation of 

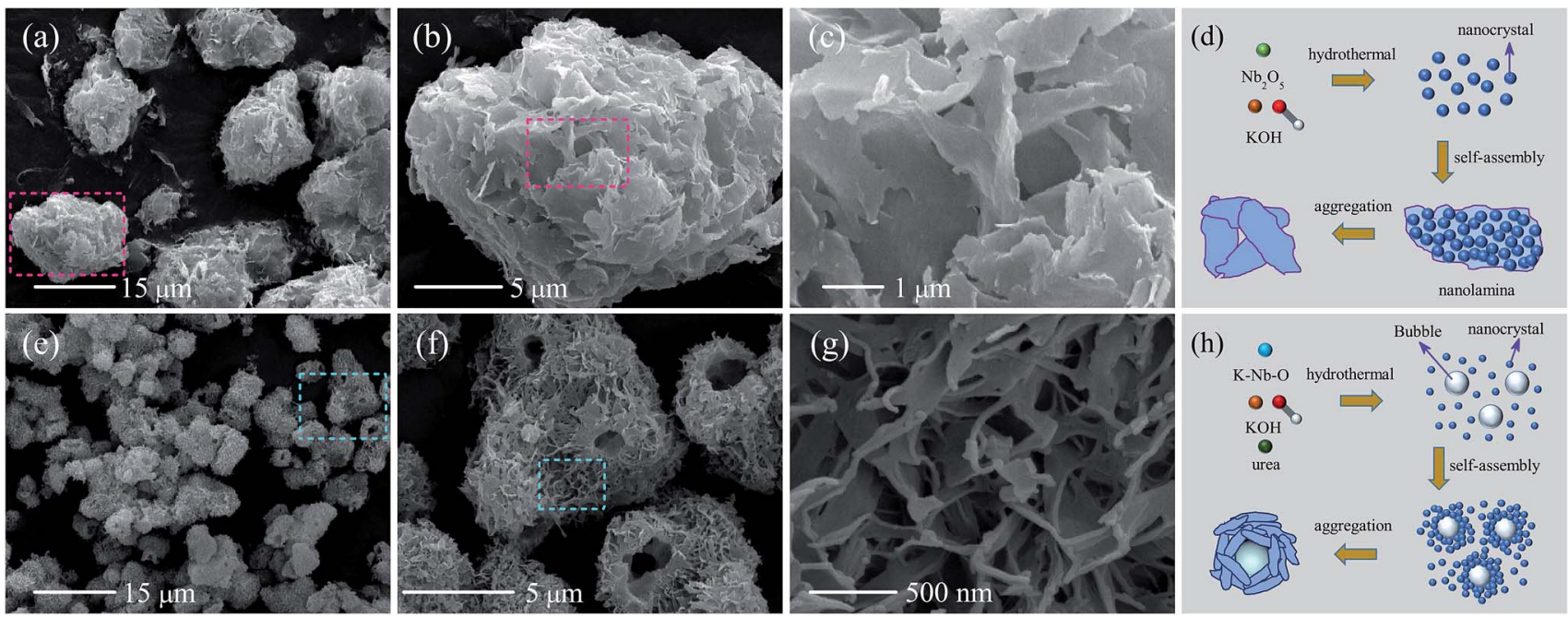

Fig. 2 SEM images of (a)-(c) KN-NL and (e)-(g) KN-NS under different magnifications. The synthesis mechanisms of (d) KN-NL and (h) KN-NS.

the nanocrystals forming compact shells around the gas bubbles. ${ }^{44}$ Moreover, as shown in Fig. S2 (ESI†ं), it can be found that only adequate urea and reaction time can synthesize the fine KN-NS.

Microstructural information of KN-NL@PbS was further characterized by transmission electron microscopy (TEM) and selected area electron diffraction (SAED). As we can see, the PbS QDs (about $2 \mathrm{~nm}$ ) were uniformly distributed on the surface of KN-NL, as shown in Fig. 3(a) and (b). The SAED patterns (shown
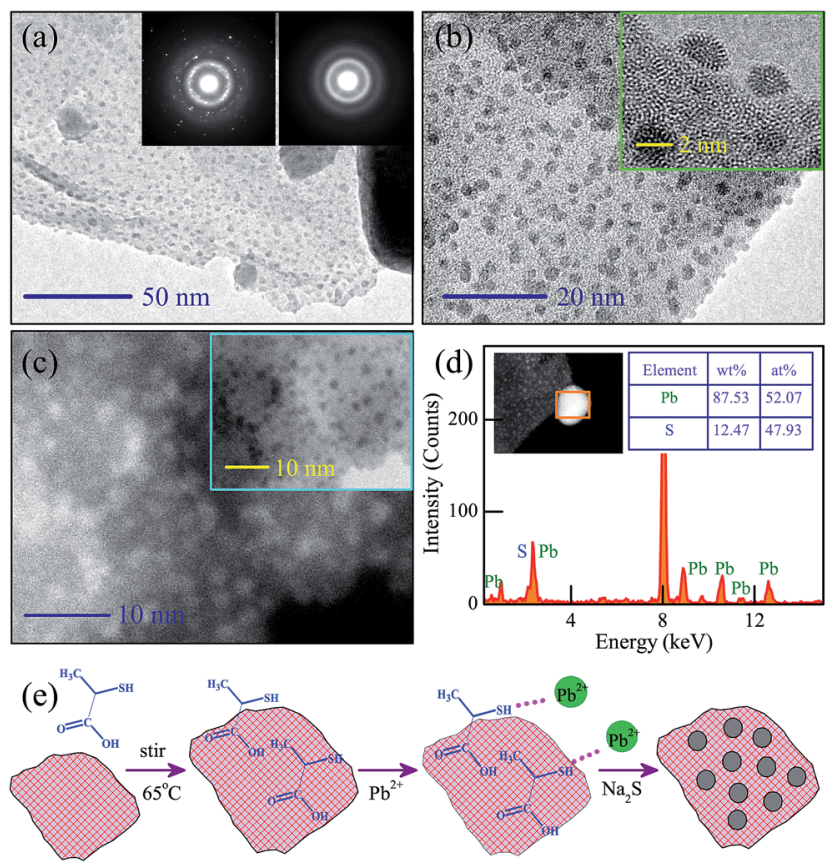

Fig. 3 TEM images of (a) and (b) KN-NL(aPbS under different magnifications. Note that the inset in (a) shows the SAED patterns of KN$\mathrm{NL}(\mathrm{PbS}$ and PbS, respectively. (c) TEM images of the bright and dark contrast of different elements. (d) The EDS spectrum of PbS and the inset showing the proportion of $\mathrm{Pb}$ and $\mathrm{S}$ elements. (e) The synthesis mechanism of $\mathrm{KN}-\mathrm{NL}(\mathrm{aPbS}$. in the inset of Fig. 3(a)) further prove the mixed-phase nature of single crystal KN (bright diffraction spots) and the massive accumulation of $\mathrm{PbS}$ (diffraction rings). To prove the existence of $\mathrm{PbS}$ (black dots), Fig. 3(c) and its inset provide the bright and dark contrast. The phenomenon can be ascribed to the fact that $\mathrm{Pb}$ has a larger atomic number than others. In the energy dispersive X-ray spectroscopy (EDS) spectrum shown in Fig. 3(d), peaks attributed to $\mathrm{S}$ and $\mathrm{Pb}$ were observed, and the atomic ratio is about $13: 12$, which is approximate to an ideal stoichiometric value. This confirmed that the obtained product was composed of KN loaded with PbS QDs. The synthesis process of KN-NL@PbS is shown in Fig. 3(e). The surfacefunctionalized thiolactic acid linker was used to generate the PbS QDs. The carboxylic group of thiolactic acid reacts with the surface of KN-NL and leaves the thiol group to combine with $\mathrm{Pb}^{2+}$. The growth of $\mathrm{PbS}$ nanocrystals occurs after adding the $\mathrm{Na}_{2} \mathrm{~S}$ solution.

\subsection{BET, DSC, CV and EIS analysis}

As an effective photocatalytic material, the specific surface area and pore-size distribution of KN are two key factors that affect its catalytic abilities. As shown in Fig. 4(a) and (c) and S3 (ESI $\dagger$ ), the BET specific surface areas of KN-NL, KN-NS, and P25 $\mathrm{TiO}_{2}$ are about 33.6, 94.9 and $53.4 \mathrm{~m}^{2} \mathrm{~g}^{-1}$, respectively, and all samples exhibit a characteristic isotherm of type IV with H3type hysteresis loops, suggesting the existence of a mesoporous structure. As expected, KN-NS with a hollow sphere structure provides a relatively larger BET surface area. The mean pore diameter of both KN-NL and KN-NS is approximately $4 \mathrm{~nm}$ with a narrow pore size distribution, as shown in the inset of Fig. 4(a) and (c). It has been extensively reported that materials with a high BET surface area would be excellent photocatalysts. ${ }^{6,24}$ Therefore, KN-NL and KN-NS were expected to exhibit high photocatalytic activity.

The stability was further confirmed by the TGA-DSC data of KN-NL and KN-NS, as shown in Fig. 4(b) and (d). It can be seen 

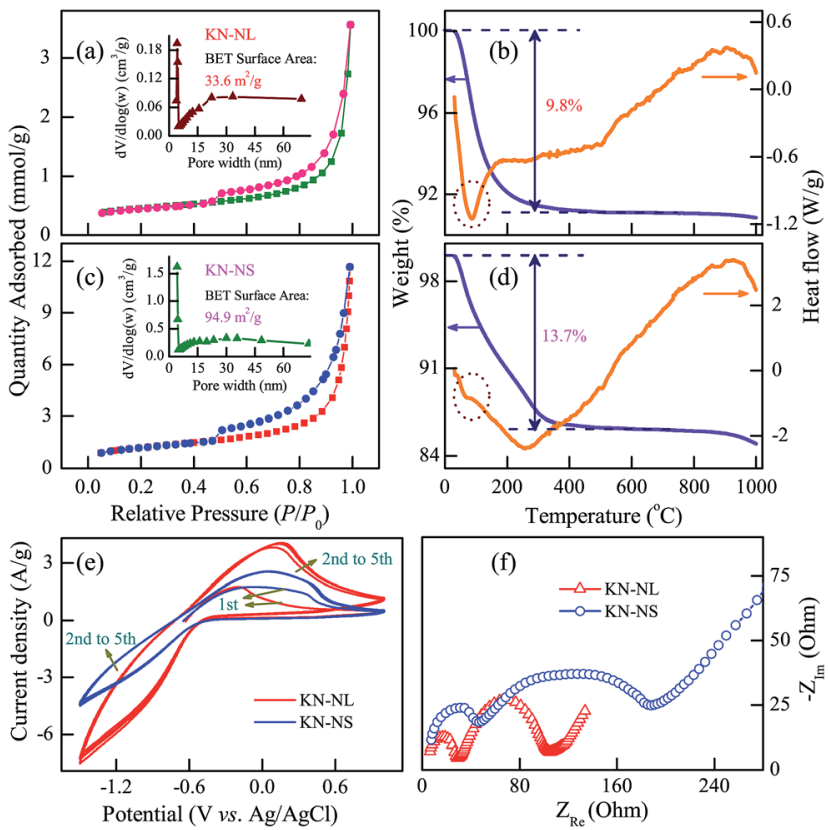

Fig. 4 The nitrogen adsorption-desorption isotherm and pore-size distribution (inset) for (a) KN-NL and (c) KN-NS. TGA-DSC curves of (b) KN$\mathrm{NL}$ and (d) KN-NS. (e) CV curves and (f) EIS Nyquist plots of KN-NL and KN$\mathrm{NS}$ in an aqueous solution of $0.1 \mathrm{~mol} \mathrm{~L}^{-1} \mathrm{HCl}$ based three-electrode test.

that the TGA-DSC curves of KN-NS are similar to those of KNNL. Both samples exhibit one distinctive weight loss stage with an endothermic peak, which takes place in the temperature range of $30-400{ }^{\circ} \mathrm{C}$ due to the evaporation of adsorbed water. The total mass losses of $\mathrm{KN}-\mathrm{NL}$ and $\mathrm{KN}-\mathrm{NS}$ were $9.8 \%$ and $13.7 \%$, respectively. Compared with $\mathrm{KN}-\mathrm{NL}$, there is more weight loss in KN-NS. This can be attributed to the hollow sphere structure of KN-NS and its larger BET surface area, which can bring about more molecules to adsorb on its surface. There are no distinct exothermal or endothermic peaks found in the corresponding DSC curves over the temperature range of 400$1000{ }^{\circ} \mathrm{C}$, which implies no phase transition for either of the two samples below $1000^{\circ} \mathrm{C}$. As a result, it can be concluded that $\mathrm{KN}$ NL and KN-NS are quite thermo-stable under high temperatures lower than $1000{ }^{\circ} \mathrm{C}$, which extends the range of their applications.

Generally, the cyclic voltammetry (CV) and electrochemical impedance spectra (EIS) technique based three-electrode test has been frequently adopted to investigate the charge transfer process. ${ }^{45}$ As shown in Fig. 4(e), it can be found that KN-NL shows enhanced current density compared with KN-NS, which indicates an improved electron transfer rate and separation efficiency of the photoinduced carriers. In addition, both KN-NL and KN-NS show two semicircles and a slash according to EIS Nyquist plots (shown in Fig. 4(f)). The arc radius of the EIS Nyquist plot of KN-NL is smaller than that of KN-NS, suggesting that KN-NL has a decrease in the solid-state interface layer resistance, adsorption resistance and the charge-transfer resistance on the surface. The $\mathrm{CV}$ and EIS results indicate that $\mathrm{KN}$ NL might show better photocatalytic performance than KN-NS.

\subsection{Adsorption and photodegradation activities for dyes}

Dye adsorption and degradation is an interesting topic for efficient treatment of dye effluents. ${ }^{46-50}$ In this work, rhodamine $\mathrm{B}(\mathrm{RhB})$ and methylene blue (MB) dyes were adopted as representative organic pollutants to evaluate the adsorption and photodegradation performances of $\mathrm{KN}$. Before light irradiation, the mixed dye suspensions were stirred in the dark for $1 \mathrm{~h}$ to establish an adsorption-desorption equilibrium in order to investigate the adsorption effects. The adsorption and photodegradation efficiency of the samples is defined as $\left(C_{0}-C\right) / C_{0}$, where $C_{0}$ and $C$ denote the initial concentration and the realtime concentration of the $\mathrm{RhB}$ or $\mathrm{MB}$ aqueous solutions, respectively. It is believed that $\mathrm{pH}$ is an important factor that influences the adsorption and photocatalytic performance of catalysts. As evident in Fig. 5(a) and (c), both the adsorption and degradation efficiency of $\mathrm{KN}-\mathrm{NL}$ and $\mathrm{KN}-\mathrm{NS}$ dramatically increased with the decreasing $\mathrm{pH}$ values. At the $\mathrm{pH}$ values of 5 , 7, 9 and 11, the average adsorption efficiency of KN-NL was only about $1 \%$ after $60 \mathrm{~min}$. However, when the $\mathrm{pH}$ value was decreased to 3 , the efficiency was about $60 \%$. A similar phenomenon was found with KN-NS. Dramatically, the adsorption efficiency of KN-NS is approximately $96 \%$ when the $\mathrm{pH}=3$. Blank experiments without a catalyst show negligible adsorption and photocatalytic activities, which indicates that the degradation reactions are truly driven by a photocatalytic process. Generally, the photodegradation rate of pollutants is often expressed by the Langmuir-Hinshelwood $(\mathrm{L}-\mathrm{H})$ equation, which can be expressed as $\ln \left(C_{0} / C\right)=k t+A$, where $k$ denotes the photodegradation rate and $t$ is the reaction time. The $k$ values can be obtained from the slope of the linear plots of $\ln \left(C_{0} / C\right)$ versus $t$. As shown in Fig. 5(b) and (d), the photodegradation process can be well fitted into a pseudo-first order reaction. As an example, the $k$ value of $\mathrm{KN}-\mathrm{NL}$ is $0.193 \mathrm{~min}^{-1}$ when the $\mathrm{pH}=$ 3. In consideration of the preeminent adsorption ability, we simultaneously present the color contrast of KN-NL and KN-NS before and after centrifugation of the RhB dye solution in Fig. S4(b) and (d) (ESI $\dagger$ ). As an example, when the value of the $\mathrm{pH}$ was 3 , the color of the KN-NL suspension changed with increasing irradiation time and the catalyst could return to its initial color, indicating the successful removal of RhB by photodegradation with the aid of adsorption. Although the KN-NS displays preeminent adsorption ability when $\mathrm{pH}=3$, it needs more time to completely photodegrade $\mathrm{RhB}$ compared to $\mathrm{KN}$ NL. So it can be concluded that KN-NL shows more excellent adsorption and photodegradation features in the $\mathrm{pH}$ range of 311, compared to KN-NS. In addition, the adsorption and photocatalysis experiments for $\mathrm{KN}$ have been carried out at a lower temperature. The results (not shown) indicate that the temperature of the dye solution can affect the photocatalytic activity of $\mathrm{KN}$. When the temperature is $\sim 2{ }^{\circ} \mathrm{C}, \mathrm{KN}-\mathrm{NL}$ also shows good photocatalytic performance.

In the following study, more experiments were designed to further evaluate the adsorption and photodegradation performances of KN-NL. As shown in Fig. 5(e) and (f), commercial catalyst $\left(\mathrm{TiO}_{2}\right.$, Degussa P25) and $\mathrm{H}^{+}$ion exchanged $\mathrm{KN}(\mathrm{KN}-\mathrm{NL}-$ $\mathrm{H}^{+}$) prepared in this work were used as photocatalytic reference 

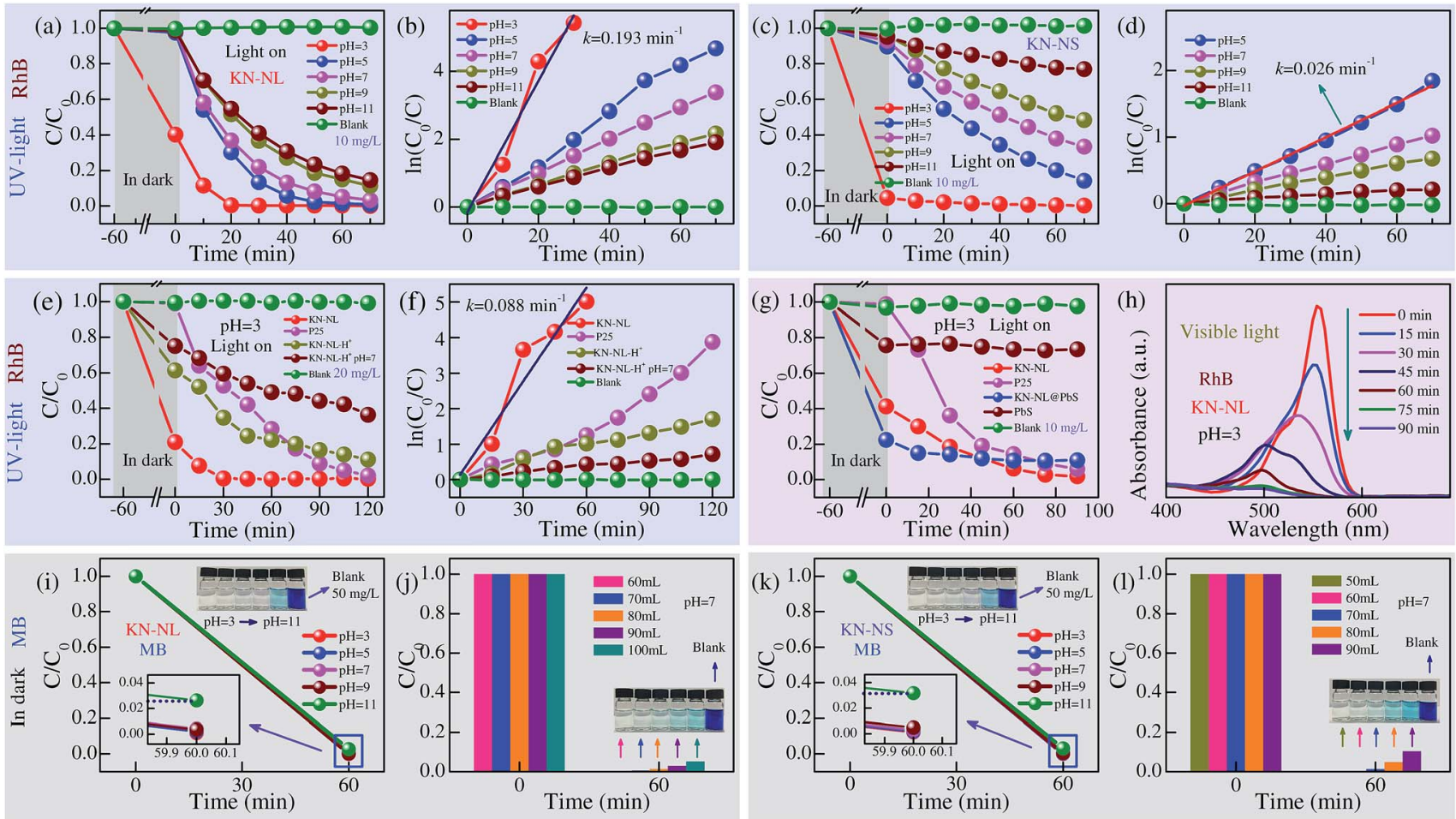

Fig. 5 The adsorption (in dark) and photodegradation (under UV-light irradiation) effects $\left(C / C_{0}\right)$ of (a) KN-NL and (c) KN-NS in the RhB aqueous solution $\left(10 \mathrm{mg} \mathrm{L}^{-1}\right.$ ) with different $\mathrm{pH}$ values. The plots of $\ln \left(C_{0} / C\right)$ versus irradiation time $t$ for (b) KN-NL and (d) KN-NS. (e) The adsorption (in dark) and photodegradation (under UV-light irradiation) effects $\left(C / C_{0}\right)$ of $\mathrm{KN}-\mathrm{NL}, \mathrm{P} 25, \mathrm{KN}-\mathrm{NL}-\mathrm{H}^{+}$and $\mathrm{KN}-\mathrm{NL}-\mathrm{H}^{+}(\mathrm{pH}=7)$ in the RhB aqueous solution (20 $\mathrm{mg} \mathrm{L}^{-1}$ ) when $\mathrm{pH}=3$, with ( $\mathrm{f}$ ) the plots of $\ln \left(C_{0} / C\right)$ versus $t$. $(\mathrm{g})$ The adsorption (in dark) and photodegradation (under VIS-light irradiation) effects $\left(C / C_{0}\right)$ of $\mathrm{KN}-\mathrm{NL}, \mathrm{P} 25, \mathrm{KN}-\mathrm{NL}\left(\mathrm{aPbS}, \mathrm{PbS}\right.$ in the RhB aqueous solution $\left(10 \mathrm{mg} \mathrm{L}^{-1}\right)$ when $\mathrm{pH}=3$. (h) The corresponding adsorption spectra of an aqueous solution of RhB at an irradiation time of 0-90 min. Note that the solid lines denote the data fitting curves. The adsorption effects ( $C / C_{0}$ ) of (i) $\mathrm{KN}-\mathrm{NL}$ and (k) KN-NS for the MB aqueous solution ( $50 \mathrm{mg} \mathrm{L}^{-1}$ ) with different $\mathrm{pH}$ values. The adsorption capacity of (j) KN-NL and (l) $\mathrm{KN}-\mathrm{NS}$ for the $\mathrm{MB}$ solution $\left(50 \mathrm{mg} \mathrm{L}^{-1}, 50-100 \mathrm{~mL}\right.$ ) when $\mathrm{pH}=7$. The insets show the color contrast of the relevant $\mathrm{MB}$ dye solution.

materials to qualitatively compare the photocatalytic ability and account for the catalytic mechanisms. A double concentration of RhB solution ( $20 \mathrm{mg} \mathrm{L}^{-1}$ ) was used to implement the UV-light photocatalysis experiments, and the $\mathrm{pH}$ values of all of the suspensions were adjusted to 3. As we can see, P25 shows negligible adsorption activities although it has a relatively larger BET surface area. KN-NL- $\mathrm{H}^{+}$did not show the same adsorption ability as KN-NL. Abnormally, when the concentration of the $\mathrm{RhB}$ solution is increased by two times, the adsorption efficiency of KN-NL is about $80 \%$, which is higher than that of $\mathrm{KN}$ $\mathrm{NL}$ at a concentration of $10 \mathrm{mg} \mathrm{L}^{-1}$. A similar phenomenon can be found in other concentrations, as shown in Fig. S5 (ESI†). A rational mechanism is described as follows. The RhB molecule will be ionized under low $\mathrm{pH}$ conditions. Due to the electrostatic attraction and hydrogen bonds, KN-NL will adsorb the RhB molecule onto/into KN-NL until the charge balances. Thus, the mixed solution of KN-NL and RhB has an adsorption equilibrium under a specific concentration. For the adsorption efficiency of KN-NL, which is defined as $\left(C_{0}-C\right) / C_{0}$, when $C_{0}$ is increased by two times, $C$ slightly increases. So the adsorption efficiency is larger than that of the one with the lower concentration. Evidently, KN-NL has the best catalytic activity compared with others in high concentrations of RhB solution. As an example, the $k$ value of KN-NL is $0.088 \mathrm{~min}^{-1}$, which is approximately half of that of KN-NL at a concentration of $10 \mathrm{mg}$
$\mathrm{L}^{-1}$. In addition, Fig. S6 (ESI $\dagger$ ) shows photocatalytic mineralization rates of the RhB solution. KN-NL exhibits a maximum value. Due to the existence of an organic intermediate after the photocatalytic process, the mineralization rates are lower than the corresponding photodegradation rates, and they may be underestimated due to introduction of carbon dioxide or other dissociative carbon during the experimental process.

KN-NL shows superior adsorption and photodegradation performances under the low $\mathrm{pH}$ value for $\mathrm{RhB}$ solution, however, these photocatalysis experiments were carried out under UV-light irradiation. It is of great significance to investigate the VIS-light driven photocatalytic activity in order to widen the practical applications of KN nano-materials. As shown in Fig. $5(\mathrm{~g})$, the pure $\mathrm{PbS}$ with weak adsorption properties shows negligible photodegradation performance under VIS-light irradiation. The adsorption activities of KN-NL@PbS were enhanced with the aid of PbS QDs. However, the composite did not show the expected photocatalysis performance under VISlight irradiation. This might be attributed to the fast recombination of photo-induced carriers. ${ }^{34}$ The photo-induced carriers cannot effectively transfer into KN-NL. Moreover, KN-NL@PbS has limited ability to absorb visible light due to the amount of anchored PbS QDs being limited. Thus, it did not show good photocatalytic performance. Our work indicates that PbS QDs might not be a good modifier to improve the photocatalytic 
performance of KN-NL. Noticeably, KN-NL displays the best photocatalytic activity in spite of the wide band gap. Fig. 5(h) shows the adsorption spectra of RhB solution in the presence of $\mathrm{KN}-\mathrm{NL}$. It was found that the main absorbance of the degraded solution ( $\sim 553 \mathrm{~nm})$ has a significant reduction and a blue shift, which could be attributed to the gradual formation of a series of $\mathrm{N}$-de-ethylated intermediates. Note that due to the different experimental conditions, such as the kind of dye, the concentration of dye and the mass of catalyst, it is hard to tell which is the better one when comparing with previous reports. However, most of the previous reports have obviously ignored the ability of adsorbing dyes and the influence derived from the $\mathrm{pH}$ of the dye solution. Remarkably, the KN-NL prepared in our work shows good photocatalytic activity.

As we know, both RhB and MB dyes are classic organic dye pollutants. In this work, the $\mathrm{KN}$ nano-materials show superior adsorption performance for RhB dye. It was believed that they would present a better adsorption ability for MB dye which has a smaller molecular size. Fig. 5(i) and (k) show the adsorption effects $\left(C / C_{0}\right)$ of $\mathrm{KN}-\mathrm{NL}$ and $\mathrm{KN}-\mathrm{NS}$, respectively, for the $\mathrm{MB}$ aqueous solution ( $50 \mathrm{mg} \mathrm{L}^{-1}$ ) with different $\mathrm{pH}$ values. It was found that the initial $\mathrm{pH}$ of the suspension solution plays a key role in the adsorption of $\mathrm{MB}$ dye. At the $\mathrm{pH}$ values of 3, 5, 7 and 9, the adsorption efficiencies of both KN-NL and KN-NS were approximately $100 \%$ after $60 \mathrm{~min}$. When increasing the $\mathrm{pH}$ value to 11 , the adsorption efficiency of both of the samples dramatically decreased by $2-3 \%$, accompanied by a color deepening. Fig. 5(j) and (l) show the adsorption capacity of KN-NL (20 mg) and KN-NS (20 mg), respectively, for the MB solution (50 $\mathrm{mg} \mathrm{L}^{-1}, 50-100 \mathrm{~mL}$ ) when the $\mathrm{pH}$ value was 7 . It can be seen that the maximum adsorption capacities of $\mathrm{KN}-\mathrm{NL}$ and $\mathrm{KN}-\mathrm{NS}$ were $70 \mathrm{~mL}$ and $60 \mathrm{~mL}$, respectively, which is equal to $175 \mathrm{mg} \mathrm{g}^{-1}$ (dye/sample) and $150 \mathrm{mg} \mathrm{g}^{-1}$, respectively. The inset color contrast in Fig. S7(a) (ESI $\dagger$ ) indicates that $\mathrm{P} 25$ shows negligible adsorption activity for $\mathrm{MB}$ in the $\mathrm{pH}$ range of $3-11$.

\subsection{Relevant kinetics mechanisms}

It is of great significance to investigate the relevant kinetics mechanisms in order to further guide the photocatalytic performance of these semiconductors and their further application as photocatalysts. The band gap structure of the semiconductor plays a key role in the enhancement of photocatalytic activity. UV-VIS diffuse reflectance spectra (DRS) of KN-NL, KNNS and $\mathrm{KN}-\mathrm{NL}-\mathrm{H}^{+}$are illustrated in Fig. 6(a). All spectra clearly show the characteristic absorption edge. The band gaps $\left(E_{\mathrm{g}}\right)$ of $\mathrm{KN}-\mathrm{NL}, \mathrm{KN}-\mathrm{NS}$ and $\mathrm{KN}-\mathrm{NL}-\mathrm{H}^{+}$are estimated to be 3.74, 3.81 and $3.47 \mathrm{eV}$, respectively, from $(A h \nu)^{2} v s$. $h \nu$ plots (inset in Fig. 6(a)). Although the $E_{\mathrm{g}}$ of $\mathrm{KN}-\mathrm{NL}$ is larger than that of $\mathrm{KN}-\mathrm{NL}-\mathrm{H}^{+}, \mathrm{KN}-$ $\mathrm{NL}-\mathrm{H}^{+}$shows poorer adsorption and photocatalytic activities. This indicates that the reason for the superior adsorption and photodegradation performances of $\mathrm{KN}-\mathrm{NL}$ does not originate from the production of $\mathrm{KN}-\mathrm{NL}-\mathrm{H}^{+}$in dye solution with a low $\mathrm{pH}$. In addition, the above $\mathrm{CV}$ and EIS analysis results confirmed the improved electron transfer rate and decreased charge transfer resistance for KN-NL, as compared with KN-NS.
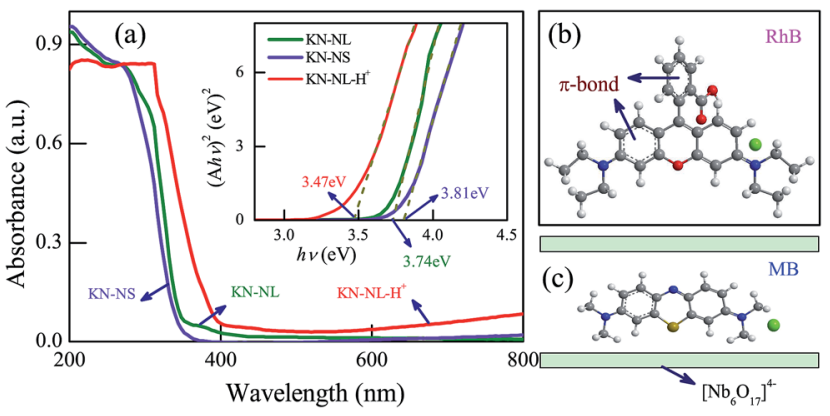

(d)

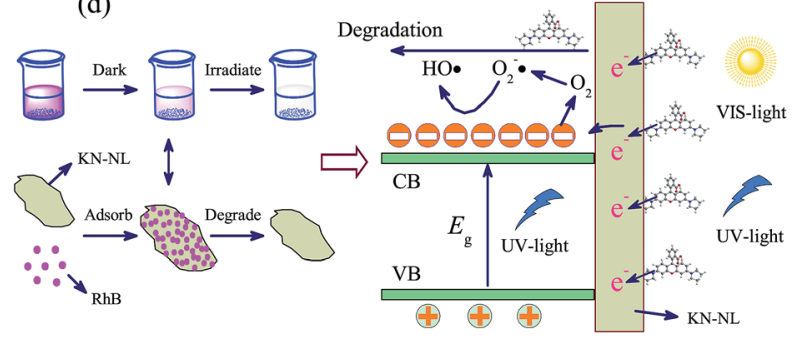

Fig. 6 (a) UV-VIS DRS of KN-NL, KN-NS and $\mathrm{KN}-\mathrm{NL}-\mathrm{H}^{+}$with the inset curve of $(A h \nu)^{2} v s$. $h \nu$. The molecular structure of (b) RhB and (c) MB. (d) The relevant kinetics mechanisms of adsorption and photocatalysis for $\mathrm{KN}-\mathrm{NL}$ in RhB solution.

It is imperative to understand the mechanisms of adsorption and photocatalysis. Fig. 6(b) and (c) show the molecular structure of $\mathrm{RhB}$ and $\mathrm{MB}$, respectively. It was found that the molecular size of $\mathrm{RhB}$ is larger than MB. The adsorption effects of the as-synthesized samples for $\mathrm{MB}$ dye can be attributed to the interspace of the layered $\mathrm{KN}$ and the electrostatic attraction. Moreover, both of the two dyes have a $\pi$-bond, which can excite electrons by light irradiation due to the gap between the HOMO and LUMO orbitals. Therefore, more photo-induced electrons can be injected into the conduction bands of $\mathrm{KN}$. It is believed that the relationship between KN-NL and dyes involves electrostatic attraction and hydrogen bonds, which can make the dye molecule absorb onto/ into the KN-NL. Moreover, the dyes absorbed onto/into the KN-NL can be in situ degraded rapidly under light irradiation. Due to the high adsorption ability of the KN-NL towards dyes and the concentration difference between these two interface layers, the decomposed dyes could be replenished continuously with dyes until complete degradation. Note that under low $\mathrm{pH}$ condition, the KN-NL also shows good photodegradation performance under VISlight irradiation, which may be due to the effects of dye sensitization, just like $\mathrm{TiO}_{2}$. The mechanism is vividly depicted in Fig. 6(d). Meaningfully, the mechanism by which the adsorption and photodegradation performances of $\mathrm{KN}$ are strongly affected by the initial $\mathrm{pH}$ of dye solution can be helpful in exploring the photocatalytic activities of other similar layered materials. In addition, in consideration of the superior adsorption and photodegradation performances of KN-NS and KN-NL, as shown in Fig. S8 (ESI†), we designed a highly-efficient path to deal with dye wastewater.

\subsection{Energy storage applications}

To investigate the potential energy storage applications of $\mathrm{KN}$, we assembled a two-electrode coin-cell using KN-NL as the 
anode material for the LIBs. Fig. 7(a) shows the typical cyclic voltammetry (CV) curves of KN-NL for the initial six cycles in the voltage range of $0.02-3.0 \mathrm{~V}$ at a scan rate of $0.5 \mathrm{mV} \mathrm{s}^{-1}$. As we can see, the reduction peaks are observed at around 0.52, 0.95 and $1.41 \mathrm{~V} v s . \mathrm{Li}^{+} / \mathrm{Li}$ during the first reduction process $(\mathrm{Li}$ insertion), and the oxidation peaks are observed at about $0.77 \mathrm{~V}$ $v s . \mathrm{Li}^{+} / \mathrm{Li}$ during the first oxidation process (Li extraction). The subsequent curves show quite good reproducibility. Rate performance is one of the most important characteristics for evaluating LIBs. As shown in Fig. 7(b), after undergoing a high current density of $1600 \mathrm{~mA} \mathrm{~g}^{-1}$, a specific capacity of $109 \mathrm{~mA} \mathrm{~h}$ $\mathrm{g}^{-1}$ can be maintained when the current density comes back again to $50 \mathrm{~mA} \mathrm{~g}^{-1}$, which suggests excellent reversibility and cyclic stability of the KN-NL electrode. Correspondingly, Fig. 7(c) presents a typical galvanostatic charge-discharge (GCD) profile of the KN-NL electrode at different current densities within the range of $50-800 \mathrm{~mA} \mathrm{~g}^{-1}$. Obviously, with increasing charge-discharge current densities, the specific capacity of KN-NL decreases gradually. Moreover, the cycling performance was further evaluated at a current density of 200 $\mathrm{mA} \mathrm{g}^{-1}$, as shown in Fig. 7(d). Although some capacity loss occurred after 200 cycles, the KN-NL electrode still maintained a discharge capacity of about $72 \mathrm{~mA} \mathrm{~h} \mathrm{~g}{ }^{-1}$, corresponding to about $86 \%$ of that at the 10 th cycle (about $84 \mathrm{~mA} \mathrm{~h} \mathrm{~g}^{-1}$ ), and the coulombic efficiency was very high (around 99.5\%). Fig. 7(e) displays the GCD curves in the 1st, 2nd, 100th and 200th cycles of the KN-NL electrode, which were measured at a current density of $200 \mathrm{~mA} \mathrm{~g}^{-1}$ in the voltage range of $0.02-3.0 \mathrm{~V}$. As can be seen, in the first cycle, the KN-NL reveals a considerable discharge specific capacity of about $513 \mathrm{~mA} \mathrm{~h} \mathrm{~g}{ }^{-1}$. Three inconspicuous plateaus at around $1.41,0.95$ and $0.52 \mathrm{~V}$ are observed in the first discharge process, but in subsequent GCD curves, the plateaus disappeared gradually, which agrees well with the above CV study. While the nature of the initial irreversible capacity is still unclear, the electrolyte decomposition, destructive structure or irreversible reaction might lead to the phenomenon. In addition, the electrochemical impedance spectra (EIS) of the KN-NL electrodes after CV measurement and 200 cycles (at a current density of $200 \mathrm{~mA} \mathrm{~g}^{-1}$ ) are compared in Fig. 7(f). In the Nyquist plots, obviously, the diameter of the semicircle decreases after 200 cycles, compared with that after CV measurement, which demonstrates that KN-NL possesses the higher contact and charge transfer resistances at the original state. In order to demonstrate its application, as shown in the inset of Fig. 7(d), using a two-electrode coin-cell based on the KN-NL electrode can still lighten a white LED (about $2.9 \mathrm{~V}$, $20 \mathrm{~mA}$ ), after being cycled 200 times at a current density of 200 $\mathrm{mA} \mathrm{g}^{-1}$. In addition, KN-NS as an anode material for lithium ion batteries has been also investigated. It shows a similar phenomenon to KN-NL, as shown in Fig. S9 and S10† (ESI $†$ ).

KN shows the interesting and complicated Li insertion/ extraction reactions, however, to the best of our knowledge, there is almost no report on proposing the relevant mechanisms of lithium ion insertion into KN. In order to further understand the LIB performance of KN, as shown in Fig. 8, we have proposed possible mechanisms. As we know, $\mathrm{KN}$ is composed of octahedral units of $\mathrm{NbO}_{6}$ and possesses a typical 2D layered structure. During the first $\mathrm{Li}$ insertion process, the
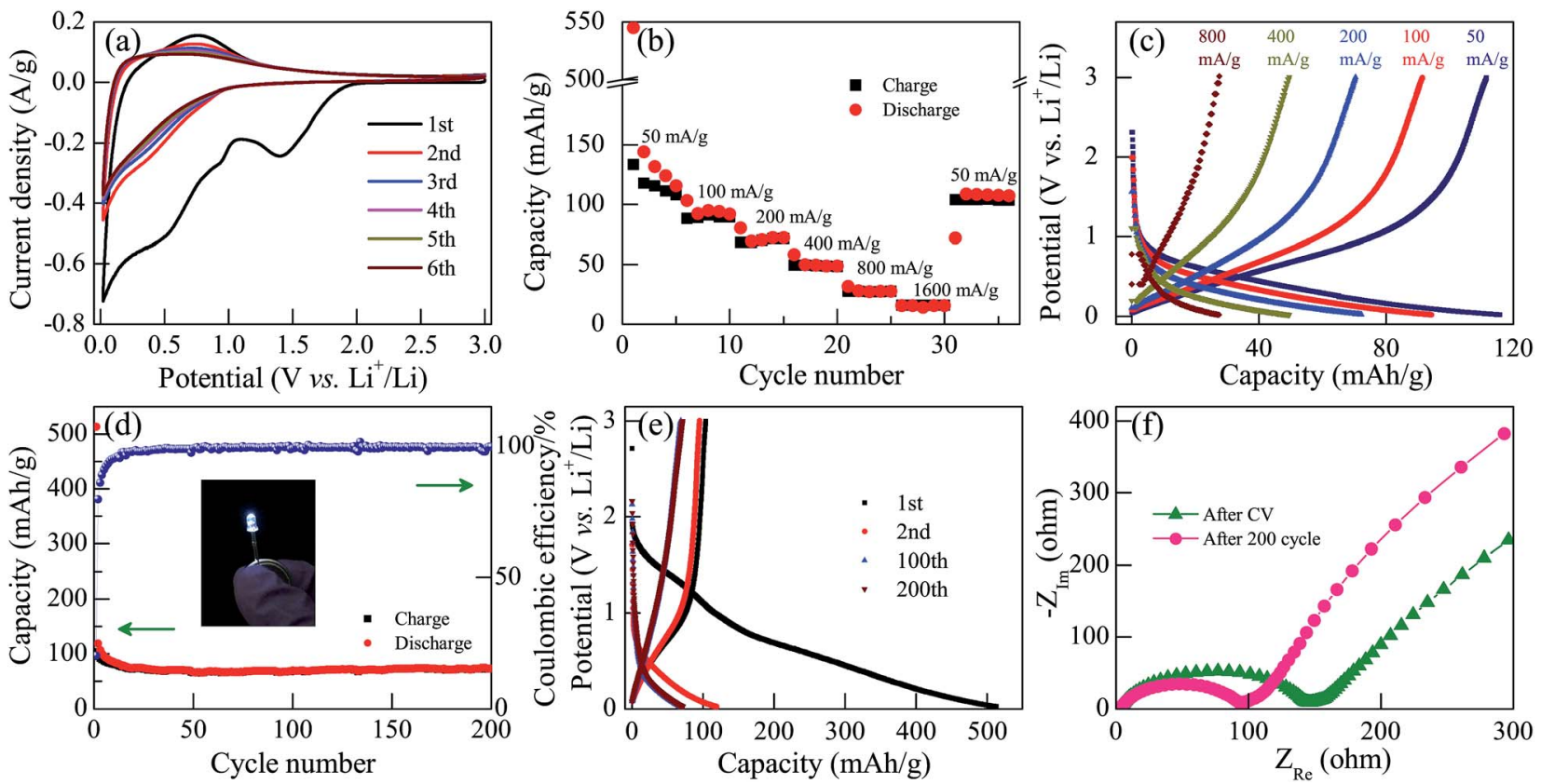

Fig. 7 (a) Cyclic voltammetry (CV) curves of $\mathrm{KN}-\mathrm{NL}$ measured at a scan rate of $0.5 \mathrm{mV} \mathrm{s}{ }^{-1}$ in the voltage range of $0.02-3.0 \mathrm{~V}\left(\mathrm{vs}\right.$. $\left.\mathrm{Li}^{+} / \mathrm{Li}\right)$. (b) The rate performance of KN-NL at different current densities. (c) Galvanostatic charge-discharge (GCD) profiles of KN-NL at different current rates. (d) The cycling stability and coulombic efficiency of KN-NL at a current density of $200 \mathrm{~mA} \mathrm{~g}^{-1}$. (e) GCD profiles of KN-NL in the $1 \mathrm{st}$, 2nd, 100 th and 200th cycles measured at a current density of $200 \mathrm{~mA} \mathrm{~g}^{-1}$. (f) Nyquist plots of KN-NL after CV measurement and 200 cycles at a current density of $200 \mathrm{~mA} \mathrm{~g}^{-1}$, in the frequency range of $100 \mathrm{kHz}$ to $0.01 \mathrm{~Hz}$. Note that the inset in (d) is the photograph of a white LED powered by a two-electrode coin-cell based on the KN-NL electrode. 


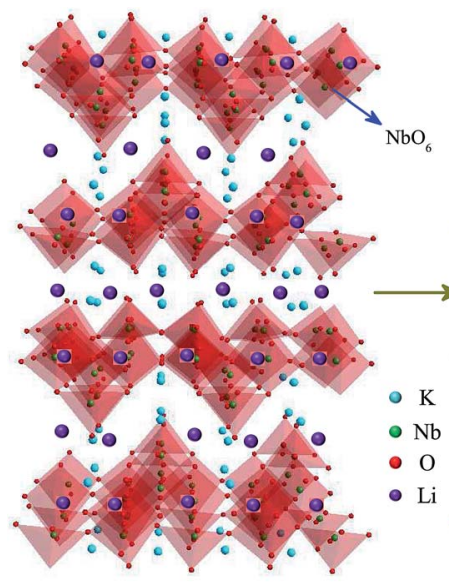

The 1st Li insertion ( $\alpha$ and $\beta$ )

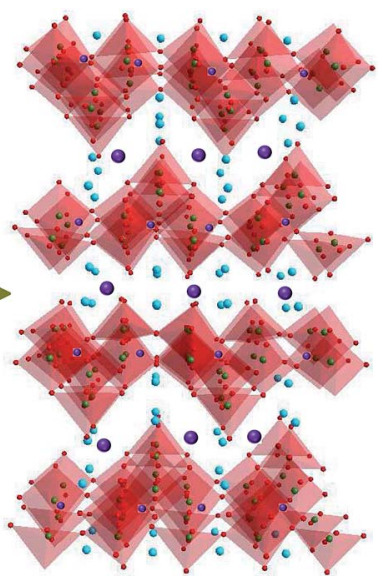

The 2 nd Li insertion $(\beta)$
Possible mechanism $\alpha$ : redox chemistry of $\mathrm{Nb} \longleftrightarrow \mathrm{Nb}^{5+}+\mathrm{e}^{-} \longrightarrow \mathrm{Nb}^{4}$ $\beta$ : solid-solution reaction $\longleftrightarrow \mathrm{KN}+\mathrm{Li}^{+}+\mathrm{e}^{-} \rightarrow \mathrm{Li}-\mathrm{KN}$

Fig. 8 The possible mechanisms of lithium ion intercalation (ignoring the actual atomic size and intercalation capacity).

reduction peak at around $1.41 \mathrm{~V} v s . \mathrm{Li}^{+} / \mathrm{Li}$ might be ascribed to the valence variation of $\mathrm{Nb}^{5+}$ to $\mathrm{Nb}^{4+}$. It disappears in the subsequent cycles, which indicates that the process is irreversible. The reduction peaks at around 0.52 and $0.95 \mathrm{Vvs.} \mathrm{Li}^{+}$/ Li might be ascribed to the complicated solid-solution reaction of $\mathrm{Li}$ and $\mathrm{KN}$. As a result, it obtains a relatively high discharge specific capacity at the first Li insertion process, but this then decreases rapidly due to the irreversible valence variation of the $\mathrm{Nb}$ element. Relevant kinetics mechanisms were depicted in Fig. 8. In addition, we speculate that the reason for the low reversible capacity might be the lack of $\mathrm{Li}^{+}$ insertion sites in the $\mathrm{KN}$ crystal structure and low electrical conductivity.

\section{Conclusion}

In conclusion, we have successfully synthesized the $\mathrm{KN}$ nanofamily by making full use of raw materials. Due to the different morphology states, the $\mathrm{KN}$ nano-family shows unique microstructures and optoelectronic properties amongst each other. Both KN-NL and KN-NS display superior adsorption and photodegradation performances, which are strongly affected by the initial $\mathrm{pH}$ of the dye solution. Based on these, we propose an effective strategy to treat dye wastewater by using KN-NL and $\mathrm{KN}-\mathrm{NS}$. In addition, $\mathrm{KN}$ has been proved to be a potential anode material for lithium ion batteries. Further studies are in progress to achieve higher capacities and better rate performances for $\mathrm{KN}$ by improving its electrical conductivity. Therefore, we conclude that our present work on the $\mathrm{KN}$ nano-family not only proposes a highly-efficient photocatalyst and strategy for dye degradation, which can be helpful in exploring the photocatalytic activities of other similar layered materials, but also might open up a novel avenue for potential multifunctional KNbased energy storage applications.

\section{Acknowledgements}

This work was financially supported by the Major State Basic Research Development Program of China (Grant No. 2013CB922300), the Natural Science Foundation of China (Grant No. 61674057, 11374097, 61376129, 61504156, and 61227902), the Projects of Science and Technology Commission of Shanghai Municipality (Grant No. 15JC1401600 and 14XD1401500), and the Program for Professor of Special Appointment (Eastern Scholar) at Shanghai Institutions of Higher Learning.

\section{References}

1 I. Grinberg, D. V. West, M. Torres, G. Y. Gou, D. M. Stein, L. Y. Wu, G. N. Chen, E. M. Gallo, A. R. Akbashev, P. K. Davies, J. E. Spanier and A. M. Rappe, Nature, 2013, 503, 509-512.

2 X. G. Yu, T. J. Marks and A. Facchetti, Nat. Mater., 2016, 15, 383-396.

3 H. Y. Zhu, Y. Wang, J. Xiao, M. Liu, S. M. Xiong, Z. J. Wong, Z. L. Ye, Y. Ye, X. B. Yin and X. Zhang, Nat. Nanotechnol., 2014, 10, 151-155.

4 L. F. Wang, S. H. Liu, Z. Wang, Y. L. Zhou, Y. Qin and Z. L. Wang, ACS Nano, 2016, 10, 2636-2643.

5 N. Miyamoto, K. Kuroda and M. Ogawa, J. Am. Chem. Soc., 2001, 123, 6949-6950.

6 C. Zhou, Y. F. Zhao, L. Shang, Y. H. Cao, L. Z. Wu, C. H. Tung and T. R. Zhang, Chem. Commun., 2014, 50, 9554-9556.

7 K. Mori, S. Ogawa, M. Martis and H. Yamashita, J. Phys. Chem. C, 2012, 116, 18873-18877.

8 N. Miyamoto and T. Nakato, Adv. Mater., 2002, 14, 12671270.

9 Y. L. Ma, X. Q. Liu, Y. Li, Y. G. Su, Z. L. Chai and X. J. Wang, J. Hazard. Mater., 2014, 279, 537-545.

10 R. Abe, M. Hara, J. N. Kondo, K. Domen, K. Shinohara and A. Tanaka, Chem. Mater., 1998, 10, 1647-1651.

11 C. H. Hu, L. H. Zhang, L. Y. Cheng, J. Chen, W. H. Hou and W. P. Ding, J. Energy Chem., 2014, 23, 136-144.

12 J. Sun, D. J. Yang, C. H. Sun, L. Liu, S. L. Yang, Y. Jia, R. S. Cai and X. D. Yao, Sci. Rep., 2014, 4, 7313.

13 Y. N. Cao, L. L. Jiang, H. F. Guo and Q. Zheng, J. Mol. Catal. A: Chem., 2014, 383-384, 209-216.

14 G. H. Du, Q. Chen, Y. Yu, S. Zhang, W. Z. Zhou and L. M. Peng, J. Mater. Chem., 2004, 14, 1437-1442.

15 X. B. Wang, J. Liu, S. Leong, X. C. Lin, J. Wei, B. Kong, Y. F. Xu, Z. X. Low, J. F. Yao and H. T. Wang, ACS Appl. Mater. Interfaces, 2016, 8, 9080-9087.

16 J. Romão and G. Mul, ACS Catal., 2016, 6, 1254-1262.

17 V. Luca, M. Osborne, D. Sizgek, C. Griffith and P. Z. Araujo, Chem. Mater., 2006, 18, 6132-6138.

18 R. Bera, S. Kundu and A. Patra, ACS Appl. Mater. Interfaces, 2015, 7, 13251-13259.

19 M. J. Li, J. Y. Wang, P. Zhang, Q. L. Deng, J. Z. Zhang, K. Jiang, Z. G. Hu and J. H. Chu, Sci. Rep., 2017, 7, 42484.

20 G. B. Saupe, T. E. Mallouk, W. Kim and R. H. Schmehl, J. Phys. Chem. B, 1997, 101, 2508-2513. 
21 K. Maeda, M. Eguchi, W. J. Youngblood and T. E. Mallouk, Chem. Mater., 2008, 20, 6770-6778.

22 M. A. Bizeto, A. L. Shiguihara and V. R. L. Constantino, J. Mater. Chem., 2009, 19, 2512-2525.

23 T. Nakato and N. Miyamoto, J. Mater. Chem., 2002, 12, 12451246.

24 G. K. Zhang, F. S. He, X. Zou, J. Gong and H. Zhang, J. Phys. Chem. Solids, 2008, 69, 1471-1474.

25 F. Madaro, R. Sæterli, J. R. Tolchard, M. A. Einarsrud, R. Holmestad and T. Grande, CrystEngComm, 2011, 13, 1304-1313.

26 S. Lee, K. Teshima, Y. Niina, S. Suzuki, K. Yubuta, T. Shishido, M. Endo and S. Oishi, CrystEngComm, 2009, 11, 2326-2331.

27 Q. L. Deng, J. Z. Zhang, T. Huang, L. P. Xu, K. Jiang, Y. W. Li, Z. G. Hu and J. H. Chu, J. Mater. Chem. C, 2015, 3, 8225-8234.

28 T. Nakato, H. Ueda, S. Hashimoto, R. Terao, M. Kameyama and E. Mouri, ACS Appl. Mater. Interfaces, 2012, 4, 4338-4347.

29 W. W. Qu, F. Chen, B. Zhao and J. L. Zhang, J. Phys. Chem. Solids, 2010, 71, 35-41.

30 M. R. Kim, Y. M. Kang and D. J. Jang, J. Phys. Chem. C, 2007, 111, 18507-18511.

31 A. Kar, S. Sain, D. Rossouw, B. R. Knappett, S. K. Pradhan and A. E. H. Wheatley, Nanoscale, 2016, 8, 2727-2739.

32 H. U. Lee, S. C. Lee, J. Won, B. C. Son, S. Choi, Y. Kim, S. Y. Park, H. S. Kim, Y. C. Lee and J. Lee, Sci. Rep., 2015, 5, 8691.

33 Y. H. Liang, M. Y. Shao, L. Liu, J. G. McEvoy, J. S. Hu and W. Q. Cui, Catal. Commun., 2014, 46, 128-132.

34 W. Q. Cui, M. Y. Shao, L. Liu, Y. H. Liang and D. Rana, Appl. Surf. Sci., 2013, 276, 823-831.

35 M. Srivastava, J. Singh, T. Kuila, R. K. Layek, N. H. Kim and J. H. Lee, Nanoscale, 2015, 7, 4820-4868.
36 J. Zhang, P. Gu, J. Xu, H. G. Xue and H. Pang, Nanoscale, 2016, 8, 18578-18595.

37 X. Yu, L. Xin, Y. Liu, W. X. Zhao, B. J. Li, X. Zhou and H. Shen, RSC Adv., 2016, 6, 27094-27101.

38 N. Nitta, D. Lei, H. R. Jung, D. Gordon, E. Zhao, G. Gresham, J. Cai, I. Luzinov and G. Yushin, ACS Appl. Mater. Interfaces, 2016, 8, 25991-26001.

39 Q. W. Lian, G. Zhou, X. H. Zeng, C. Wu, Y. H. Wei, C. Cui, W. F. Wei, L. B. Chen and C. C. Li, ACS Appl. Mater. Interfaces, 2016, 8, 30256-30263.

40 P. Arunkumar, A. G. Ashish, B. Babu, S. Sarang, A. Suresh, C. H. Sharma, M. Thalakulam and M. M. Shaijumon, RSC Adv., 2015, 5, 59997-60004.

41 L. Wang, B. Y. Ruan, J. T. Xu, H. K. Liu and J. M. Ma, RSC Adv., 2015, 5, 36104-36107.

42 C. F. Zhang, S. J. Kim, M. Ghidiu, M. Q. Zhao, M. W. Barsoum, V. Nicolosi and Y. Gogotsi, Adv. Funct. Mater., 2016, 26, 4143-4151.

43 L. T. Yan, X. H. Rui, G. Chen, W. C. Xu, G. F. Zou and H. M. Luo, Nanoscale, 2016, 8, 8443-8465.

44 X. W. Lou, L. A. Archer and Z. C. Yang, Adv. Mater., 2008, 20, 3987-4019.

45 B. Weng, X. Zhang, N. Zhang, Z. R. Tang and Y. J. Xu, Langmuir, 2015, 31, 4314-4322.

46 H. Y. Jing, T. Wen, C. M. Fan, G. Q. Gao, S. L. Zhong and A. W. Xu, J. Mater. Chem. A, 2014, 2, 14563-14570.

47 G. W. Zhan and H. C. Zeng, Chem. Mater., 2016, 28, 45724582 .

48 M. Feng, W. You, Z. S. Wu, Q. D. Chen and H. B. Zhan, ACS Appl. Mater. Interfaces, 2013, 5, 12654-12662.

49 C. H. Zhang, L. H. Ai and J. Jiang, J. Mater. Chem. A, 2015, 3, 3074-3081.

50 F. Wang, F. L. Li, M. M. Xu, H. Yu, J. G. Zhang, H. T. Xia and J. P. Lang, J. Mater. Chem. A, 2015, 3, 5908-5916. 